MATHEMATICS OF COMPUTATION

Volume 66, Number 217, January 1997, Pages 417-431

S 0025-5718(97)00800-4

\title{
ON THE KHINTCHINE CONSTANT
}

\author{
DAVID H. BAILEY, JONATHAN M. BORWEIN, AND RICHARD E. CRANDALL
}

\begin{abstract}
We present rapidly converging series for the Khintchine constant and for general "Khintchine means" of continued fractions. We show that each of these constants can be cast in terms of an efficient free-parameter series, each series involving values of the Riemann zeta function, rationals, and logarithms of rationals. We provide an alternative, polylogarithm series for the Khintchine constant and indicate means to accelerate such series. We discuss properties of some explicit continued fractions, constructing specific fractions that have limiting geometric mean equal to the Khintchine constant. We report numerical evaluations of such special numbers and of various Khintchine means. In particular, we used an optimized series and a collection of fast algorithms to evaluate the Khintchine constant to more than 7000 decimal places.
\end{abstract}

\section{INTRODUCTION}

The Khintchine constant arises in the measure theory of continued fractions. Every positive irrational number can be written uniquely as a simple continued fraction $\left[a_{0} ; a_{1}, a_{2}, \ldots, a_{n}, \ldots\right]$, i.e., with $a_{0}$ a nonnegative integer and all other $a_{i}$ positive integers. The Gauss-Kuz min distribution [13] predicts that the density of occurrence of some chosen positive integer $k$ in the fraction of a random real number is given by

$$
\operatorname{Prob}\left(a_{n}=k\right)=-\log _{2}\left[1-\frac{1}{(k+1)^{2}}\right] .
$$

In his celebrated text, Khintchine [13] uses the Gauss-Kuz'min distribution to show that for almost all positive irrationals the limiting geometric mean of the positive elements $a_{i}$ of the relevant continued fraction exists and equals

$$
K_{0}:=\prod_{k=1}^{\infty}\left[1+\frac{1}{k(k+2)}\right]^{\log _{2} k}=\prod_{k=1}^{\infty} k^{\log _{2}\left[1+\frac{1}{k(k+2)}\right]} .
$$

The fundamental constant $K_{0}$ is the Khintchine constant. Ever since Khintchine's elegant discovery there has been a keen interest in the numerical evaluation of $K_{0}$ $[14,20,26,27,24]$. It is known that this constant can be cast in terms of various converging series, the following example of which having been used by Shanks and

Received by the editor May 31, 1995, and, in revised form, February 8, 1996.

1991 Mathematics Subject Classification. Primary 11Y60, 11Y65; Secondary 11M99.

Key words and phrases. Khintchine constant, continued fractions, geometric mean, harmonic mean, computational number theory, zeta functions, polylogarithms.

Research supported by the Shrum Endowment at Simon Fraser University and NSERC. 
Wrench to provide the first high-precision numerical values for $K_{0}$ :

$$
\log \left(K_{0}\right) \log (2)=\sum_{s=1}^{\infty} \frac{\zeta(2 s)-1}{s}\left(1-\frac{1}{2}+\frac{1}{3}-\cdots+\frac{1}{2 s-1}\right) .
$$

This series can be rendered even more computationally efficient via the introduction of a free integer parameter. We used a carefully optimized free-parameter series to resolve $K_{0}$ to 7350 decimal places $\left(K_{0}=2.68545200106 \ldots\right.$ see $\left.\S 5\right)$.

The Khintchine constant can be thought of as a member of a certain class of constants we shall call Khintchine means $K_{p}$, for real numbers $p<1$. The Hölder mean of order $p$ of the continued fraction elements, namely $\lim _{k}\left[\left(a_{1}^{p}+a_{2}^{p}+\cdots+a_{k}^{p}\right) / k\right]^{1 / p}$, also exists with probability one and again with probability one equals the constant:

$$
K_{p}:=\left\{\sum_{k=1}^{\infty}-k^{p} \log _{2}\left[1-\frac{1}{(k+1)^{2}}\right]\right\}^{1 / p} .
$$

(See the final section of Khintchine's book [13] for a proof for $p<\frac{1}{2}$, or more modern references on ergodic theory for a proof for $p<1$ [19].) We may interpret $K_{0}$ as the limiting instance of the $K_{p}$ definition as $p \rightarrow 0$. We shall show in Theorem 6 below that for any negative integer $p$ the Khintchine mean of order $p$ satisfies an identity

$$
\left(K_{p}\right)^{p} \log (2)=\sum_{s=2}^{\infty}(\zeta(s-p)-1) Q_{s p},
$$

where each coefficient $Q_{s p}$ is rational. Again, there is a free-parameter generalization, which we employed to resolve the harmonic mean $K_{-1}$ also to over 7000 decimal places $\left(K_{-1}=1.74540566240 \ldots\right.$ see $\left.\S 5\right)$. It is of interest that, evidently, only $K_{0}$ can be written as a series involving exclusively even zeta arguments. The computational implications of this unique property of $K_{0}$ are discussed in $\S 5$. We should mention that aside from the series (1) for $K_{0}$ there are other previously known formulae for Khintchine means, some of which involve derivatives of the zeta function [24].

In the next section we establish the series forms (1) and (2) for $K_{0}$ and the general Khintchine means $K_{p}$, respectively. Actually, (1) and (2) can be thought of as degenerate cases of free-parameter forms in which an integer parameter can be optimized for numerical efficiency. Then in $\S 3$ we present polylogarithm series and a certain zeta-like function whose evaluations can be used to accelerate the polylogarithm series. In $\S 4$ we discuss explicit continued fractions with a view toward determining whether Hölder means exist and coincide with Khintchine means. In particular, some numbers known to have geometric mean (zeroth Hölder mean) equal to $K_{0}$ are presented. Finally, in $\S 5$ we discuss numerical details relevant to very-high-precision evaluation of Khintchine means.

\section{Fundamental identities}

This section is devoted to presenting the basic identities. We begin with a list of preliminary, largely elementary, results needed in the paper. The first lemma amounts to a set of observations due to Wrench and Shanks [27]. 
Lemma 1. (a) We have

$$
-\log (1-x) \log (1+x)=\sum_{k=1}^{\infty} \frac{A_{k}}{k} x^{2 k}
$$

where $A_{s}:=\sum_{m=1}^{2 s-1}(-1)^{m-1} / m$.

(b) Further,

$$
\sum_{k=2}^{N} \log \left(1-\frac{1}{k}\right) \log \left(1+\frac{1}{k}\right)-\sum_{k=2}^{N} \log (k-1) \log \left(1-\frac{1}{k^{2}}\right)=-\log (N) \log \left(1+\frac{1}{N}\right) .
$$

(c) Thus,

$$
\sum_{k=2}^{\infty} \log \left(1-\frac{1}{k}\right) \log \left(1+\frac{1}{k}\right)=-\log \left(K_{0}\right) \log (2) .
$$

Proof. Part (a) is most easily seen by differentiating both sides. The left-hand side becomes $f(x)-f(-x)$ where $f(x):=\log (1+x) /(1-x)$. Using the standard relationship

$$
\sum_{k=1}^{\infty} \frac{a_{k}}{1-x} x^{k}=\sum_{k=1}^{\infty}\left\{\sum_{j=1}^{k} a_{j}\right\} x^{k}
$$

produces (a).

Part (b) is easily established inductively after expanding the left-hand side.

Part (c) follows on taking limits and noting that

$$
-\sum_{k=2}^{N} \log (k-1) \log \left(1-\frac{1}{k^{2}}\right)=\log \left(K_{0}\right) \log (2),
$$

as follows from the definition of $K_{0}$.

We shall find it convenient to use the Hurwitz zeta function defined by

$$
\zeta(s, N):=\sum_{n=1}^{\infty} \frac{1}{(n+N)^{s}},
$$

so that $\zeta(s)=\zeta(s, 0)$ and so that for $N$ a nonnegative integer

$$
\zeta(s, N)=\zeta(s)-\sum_{n=1}^{N} \frac{1}{n^{s}} .
$$

With this notation we have:

Lemma 2. (a) For $N$ a positive integer,

$$
\sum_{n=2}^{\infty} \zeta(n, N)=\frac{1}{N}
$$

(b) For $N$ a positive integer,

$$
\sum_{n=1}^{\infty} \frac{\zeta(2 n, N)}{n}=\log \left(\frac{N+1}{N}\right) .
$$

(c)

$$
\int_{0}^{1} \frac{\log \left(1-t^{2}\right)}{t(1+t)} d t=-\log ^{2}(2)
$$


Proof. The proofs of the first two identities are similar and rely on expanding the zeta terms, rearranging the order of summation and re-evaluating. In both cases, the result telescopes to the desired conclusion.

Part (c) is less immediate. Actually, the indefinite integral is evaluable with the aid of the theory of the dilogarithm [15]. The integral

$$
\int_{0}^{t} \frac{\log \left(1-x^{2}\right)}{x(1+x)} d x
$$

equals the log terms

$$
-\frac{\log ^{2}(1+t)}{2}-\log ^{2}(2)+\log (2) \log (1-t)-\log (1+t) \log (1-t)+\log (t) \log (1-t)
$$

plus the dilog terms

$$
\operatorname{dilog}(t)-\operatorname{dilog}(1+t)-\operatorname{dilog}\left(\frac{1+t}{2}\right),
$$

and the limit as $t \rightarrow 1$ yields the desired result $-\log ^{2}(2)$.

We are now in a position to establish a general Shanks-Wrench identity [20] for $K_{0}$.

Theorem 3. For any positive integer $N$,

$$
\log \left(K_{0}\right) \log (2)=\sum_{s=1}^{\infty} \zeta(2 s, N) \frac{A_{s}}{s}-\sum_{k=2}^{N} \log \left(1-\frac{1}{k}\right) \log \left(1+\frac{1}{k}\right),
$$

where $A_{s}:=\sum_{m=1}^{2 s-1}(-1)^{m-1} / m$.

Remark. The integer $N$ is a free parameter that can be optimized in actual computations to significantly reduce the number of zeta evaluations required. Variation of this parameter also provides a kind of error check, for whatever the choice of positive integer $N$, one expects the same result for the left-hand side. Note that in the case $N=1$ the second summation is empty, and we recover precisely the $K_{0}$ identity (1) of $\S 1$.

Proof. Let $f(N)$ denote the right-hand side of (1). Then

$$
f(N-1)-f(N)=\sum_{s=1}^{\infty} \frac{A_{s}}{s} N^{-2 s}+\log \left(1-\frac{1}{N}\right) \log \left(1+\frac{1}{N}\right)
$$

which equals zero by Lemma 1 (a). Thus, since $\zeta(2 s, N) \rightarrow 0$ sufficiently rapidly,

$$
\begin{aligned}
f(1) & =f(N)=\lim _{N \rightarrow \infty} f(N) \\
& =-\sum_{k=2}^{\infty} \log \left(1-\frac{1}{k}\right) \log \left(1+\frac{1}{k}\right) .
\end{aligned}
$$

By Lemma 1(c), this sum agrees with $\log \left(K_{0}\right) \log (2)$.

As a companion relation to the identity of Theorem 3, we can establish an elegant integral representation for the left-hand side. There is a powerful generalization of Lemma 2(b) in the form of a generating function based on Euler's product for $\sin (\pi t) /(\pi t)$ (see [23, p. 249]). For real $t$ in $[0,1)$ define $g(t)$ by

$$
g(t):=\sum_{s=1}^{\infty} \frac{\zeta(2 s)-1}{s} t^{2 s}=-\log \left(\frac{\sin (\pi t)}{\pi t}\right)+\log \left(1-t^{2}\right),
$$


and define also the limiting case $g(1):=\log 2$. We only need observe now that, on the basis of Theorem 3 with parameter $N=1$,

$$
\log \left(K_{0}\right) \log (2)=\int_{0}^{1} \frac{\log (2)+g(t) / t}{1+t} d t,
$$

and with the help of the previous dilogarithm integral evaluation we thus arrive at an integral representation. (Reference [20] contains an equivalent integral identity.)

Corollary 4. The following integral representation holds for $K_{0}$ :

$$
\int_{0}^{1} \frac{\log [\sin (\pi t) /(\pi t)]}{t(1+t)} d t=-\log \left(K_{0}\right) \log (2) .
$$

It is amusing to observe that Lemma 1(c) may also be turned into an analogous integral form:

$$
\log \left(K_{0}\right) \log (2)=\int_{1}^{\infty} \frac{\log (\lfloor t\rfloor)}{t(1+t)} d t=\int_{0}^{1} \frac{\log (\lfloor 1 / t\rfloor)}{1+t} d t .
$$

This was observed from a very different starting point by Robert Corless [11] but follows immediately on breaking the first integral up at integer points.

We now derive new, corresponding identities for the higher-order Khintchine means. They are in some sense simpler, since one logarithmic term is replaced by a negative integral power. There is an observation that leads directly to a zeta function expansion for these general Khintchine means. Note that a sum of terms $k^{p} \log \left(1-(k+1)^{-2}\right)$ can be expressed, via expansion of the logarithm, in terms of sums of the form (note $p$ is assumed to be a negative integer):

$$
\sum_{n=2}^{\infty} \frac{1}{n^{2 s-p}(1-1 / n)^{-p}}
$$

Upon expansion of the term

$$
1 /(1-1 / n)^{-p}
$$

in powers of $1 / n$, we obtain an identity for the $p$ th power of $K_{p}$ as a series of zeta functions. The result, after the same manner of free-parameter manipulation we used for $K_{0}$, is a new series that can be thought of as a companion identity to the Shanks-Wrench expansion of Theorem 3.

Theorem 5. For negative integer $p$ and positive integer $N$ we have

$$
K_{p}^{p} \log (2)=\sum_{n=1}^{\infty} \frac{\sum_{j=0}^{\infty}\left(\begin{array}{c}
j-p-1 \\
-p-1
\end{array}\right) \zeta(2 n+j-p, N)}{n}-\sum_{k=2}^{N} \log \left(1-\frac{1}{k^{2}}\right)(k-1)^{p} .
$$

Remark. Note that for $N=1$ the final sum is empty, the coefficient of any given $\zeta(s)$ is an easily computed rational, and we immediately establish a general series with rational coefficients, (2) of $\S 1$.

Corollary 6. The constant $K_{-1}$ satisfies, for any integer $N>0$,

$$
\frac{\log (2)}{K_{-1}}=\sum_{n=1}^{\infty} \frac{\frac{1}{N}-\sum_{k=2}^{2 n} \zeta(k, N)}{n}-\sum_{k=2}^{N} \frac{\log \left(1-k^{-2}\right)}{k-1} .
$$


Proof. It suffices to show that for every positive integer $n$

$$
\sum_{j=0}^{\infty} \zeta(2 n+j+1, N)+\sum_{k=2}^{2 n} \zeta(k, N)=\frac{1}{N} .
$$

This follows immediately from Lemma 2(a).

\section{Polylogarithm Series}

There exist some interesting identities for the Khintchine constant in terms of polylogarithm evaluations. One particularly interesting polylogarithm identity is obtained by resolving the integral representation of Corollary 4 in polylogarithm terms [28]. One may employ the Euler product for $\sin z / z$ to write the integral as a sum of logarithmic integrals, each in turn expressible in terms of polylogarithms. This procedure leads to the series

$$
\log \left(K_{0}\right) \log (2)=\log ^{2}(2)+\operatorname{Li}_{2}\left(-\frac{1}{2}\right)+\frac{1}{2} \sum_{n=2}^{\infty}(-1)^{n} \operatorname{Li}_{2}\left(\frac{4}{n^{2}}\right),
$$

where, $\operatorname{Li}_{m}(z)$ is the polylogarithm function: $\operatorname{Li}_{m}(z):=\sum_{k=1}^{\infty} z^{k} k^{-m}$.

A somewhat different application of polylogarithms is to use the classical Abel identity [15]

$\log (1-x) \log (1-y)=\mathrm{Li}_{2}\left(\frac{x}{1-y}\right)+\mathrm{Li}_{2}\left(\frac{y}{1-x}\right)-\mathrm{Li}_{2}(x)-\mathrm{Li}_{2}(y)-\mathrm{Li}_{2}\left(\frac{x y}{(1-x)(1-y)}\right)$

together with Lemma $1(\mathrm{c})$, setting $x:=1 / n, y:=-1 / n$ to obtain

$$
\log \left(K_{0}\right) \log (2)=\frac{\pi^{2}}{6}-\frac{1}{2} \log ^{2}(2)+\sum_{n=2}^{\infty} \operatorname{Li}_{2}\left(\frac{-1}{n^{2}-1}\right) .
$$

An interesting line of analysis starting from this last polylogarithm series is to "peel off" parts of the $\mathrm{Li}_{2}$ function, casting the corrections in closed form. Such a procedure gives polylogarithm-based analogues of Theorem 3. For example, one can replace the last $\mathrm{Li}_{2}$ summand above with a more rapidly decaying term

$$
\operatorname{Li}_{2}\left(\frac{-1}{n^{2}-1}\right)-\frac{-1}{n^{2}-1}-\frac{1}{4} \frac{1}{\left(n^{2}-1\right)^{2}}
$$

and add back a correction

$$
-\Omega(1)+\frac{1}{4} \Omega(2)=\frac{\pi^{2}}{48}-\frac{59}{64}
$$

where $\Omega$ is the zeta-like function

$$
\Omega(m):=\sum_{n=2}^{\infty} \frac{1}{\left(n^{2}-1\right)^{m}} .
$$

Careful Eulerian partial fraction decomposition (as detailed in [7]) can be used to produce a closed-form evaluation of $\Omega(m)$ for any positive integer $m$. In this way one may accelerate the convergence of relevant polylogarithm sums. 


\section{EXPLICIT CONTINUED FRACTIONS}

It is remarkable that, even though a random fraction's limiting geometric mean exists and furthermore equals the Khintchine constant with probability one, not a single explicit real number (e.g., a real number cast in terms of fundamental constants) has been demonstrated to have elements whose geometric mean equals $K_{0}$. Likewise, for any negative integer $p$, not a single explicit real number has been shown to have elements whose Hölder mean equals $K_{p}$. In any event, it is worthwhile to mention some classical continued fractions with respect to this theoretical impasse.

The continued fraction for $e$ is

$$
e=[2 ; 1,2,1,1,4,1,1,6,1,1,8,1,1,10,1,1,12, \ldots] .
$$

The elements are eventually comprised of a meshing of two arithmetic progressions, one of which has zero common difference while the other has difference two and diverges. Thus the meshing has diverging geometric mean. Thus, $e$ does not possess geometric mean $K_{0}$. The harmonic mean for $e$ does exist, but equals $3 / 2$, which is not $K_{-1}$. It turns out that any fraction with elements lying in a single arithmetic progression can be evaluated in terms of special functions. Explicitly, for any positive integers $a, d$ we have [2, eq. 9.1.73]

$$
[a ; a+d, a+2 d, a+3 d, \ldots]=\frac{I_{a / d-1}\left(\frac{2}{d}\right)}{I_{a / d}\left(\frac{2}{d}\right)},
$$

where $I_{\nu}$ is the modified Bessel function of order $\nu$. These arithmetic progression fractions are certainly interesting, and not beyond deep analysis. It was known, for example, to C. L. Siegel that these fractions are transcendental [22]. But each such fraction has diverging geometric mean and indeed diverging Hölder means. Note that the means are monotone nondecreasing in $p$, and so a fraction with lim inf of its elements infinite has infinite means.

Another example of interest is $\pi$, whose continued fraction expansion is

$$
\pi=[3 ; 7,15,1,292,1,1,1,2,1,3, \ldots] .
$$

The continued fraction elements do not appear to follow any pattern and are widely suspected to be in some sense random. Based on the first 17,001,303 continued fraction elements, the geometric mean (of the fraction elements yielding the same precision) is 2.686393 and the harmonic mean is 1.745882 [12]. These values are reasonably close to $K_{0}$ and $K_{-1}$, but of course no conclusion can be drawn beyond this.

It is a well-known theorem of Lagrange that the elements of a simple continued fraction form an eventually periodic sequence if and only if the fraction is an irrational quadratic surd. All Hölder means for $p=0,-1,-2, \ldots$ then exist, and are completely determined by one period of elements. Hence, each Hölder mean of a quadratic surd is an algebraic number. Clearly, for any algebraic number $c=a^{1 / b}$ formed from integers $a, b$, one can write down a quadratic surd having geometric mean $c$. Along these lines, it is not hard to show that if there exists an integer $m>2$ such that

$$
\frac{\log \left(K_{0} / m\right)}{\log (2 / m)}
$$


is rational, then there exists a quadratic surd with geometric mean $K_{0}$. Thus the issue of transcendence for $K_{0}$ and related numbers is an interesting one, and one we return to in the next section.

Even though no explicit real number is known to have elements whose geometric mean is $K_{0}$, it is still possible to fabricate explicit lists of elements whose geometric mean does equal $K_{0}$. If one were in possession of some representation of $K_{0}$ to arbitrary accuracy, one could of course construct a fraction having geometric mean $K_{0}$ by appending a "2" (respectively, "3") to the element list whenever the current geometric mean were above (below) $K_{0}$. There seems to be no way to determine $a$ priori the value of, say, the $n$th element. Thus, such a constructed fraction is not explicit.

But it is possible to give an explicit list of elements having the desired property. One successful construction has been given by [1], as follows. Consider the naturally ordered rationals in $(0,1)$; that is, consider

$$
1 / 2,1 / 3,2 / 3,1 / 4,2 / 4,3 / 4,1 / 5,2 / 5,3 / 5,4 / 5, \ldots,
$$

where for each successive denominator $d=2,3,4, \ldots$ we employ in increasing order all numerators between 1 and $d-1$ inclusive. Now consider the (finite) set of fraction elements for each rational in the list. (We also demand the caveat that no such terminating fraction is allowed to end with element 1 , so for example $2 / 3$ is the fraction $[1,2]$ rather than $[1,1,1]$.) If we concatenate the elements from all the terminating fractions, the infinite chain of elements has limiting geometric mean equal to $K_{0}$. The resulting sequence of elements starts out:

$$
A=[2 ; 3,1,2,4,2,1,3,5,2,2,1,1,2,1,4,6,3,2,1,2,1,5,7,3,2, \ldots] .
$$

The geometric mean of the first 15,000 elements of $A$ is $2.35821 \ldots$, which appears low but note that as the denominator $d$ increases during construction of the elements, larger and larger elements (such as $d$ itself) appear.

But one may construct elements whose geometric mean converges much more rapidly to $K_{0}$. One such construction is based on a deterministic stochastic sampling of the Gauss-Kuz'min density, and proceeds as follows. First, for non-negative integer $n$ define the van der Corput discrepancy sequence [16] to be a set of the base2 numbers

$$
d(n)=0 . b_{0} b_{1} b_{2} \ldots,
$$

where the $b_{i}$ are the binary bits of $n$, with $b_{0}$ being least significant. As $n$ runs through positive integers, the sequence of $d(n)$ is confined to $(0,1)$ and has appealing pseudorandom properties. The construction of the number we shall call $Z_{2}$ then starts with $a_{0}:=0$, and loops as follows:

For $n=1$ to $\infty$, set $a_{n}:=\left\lfloor 1 /\left(2^{d(n)}-1\right)\right\rfloor$.

The continued fraction elements $a_{n}$ thus determined start out

$$
Z_{2}=[0 ; 2,5,1,11,1,3,1,22,2,4,1,7,1,2,1,45,2,4,1,8,1,3,1,14,1, \ldots] .
$$

On the idea that the discrepancy sequence is in a certain sense equidistributed, we are moved to posit:

Conjecture. The geometric mean of the number $Z_{2}$ is in fact the Khintchine constant $K_{0}$. Furthermore, every limiting $p$-th Hölder mean of $Z_{2}$ for $p=-1,-2, \ldots$ is the respective Khintchine mean $K_{p}$. 
With regard to the above conjecture, S. Plouffe [18] has reported a computation of the geometric and harmonic means through 5206016 continued fraction elements of $Z_{2}$. His results are 2.6854823207 and 1.7454074435 , respectively, which are remarkably close to the expected theoretical values. The authors have also been informed by $\mathrm{T}$. Wieting that he has an unpublished proof of the basic conjecture, i.e., that the limiting Hölder means of $Z_{2}$ exist for $p=0,-1,-2, \ldots$ and furthermore equal the corresponding Khintchine means [25]. In the wake of such positive results, one can ask in addition for, say, the rate of convergence to $K_{0}$. Again on the basis of the distribution of discrepancy values we think it reasonable to conjecture that the geometric mean $G_{n}$ of the first $n$ elements of $Z_{2}$ satisfies

$$
\left|G_{n}-K_{0}\right|<c / n^{1 / 2}
$$

for some absolute constant $c$.

Yet a third, and novel construction runs as follows. First, for the correct GaussKuz'min density of 1's, namely $p_{1}=\log _{2}(4 / 3)$, generate a list of 0 's and 1 's by assigning element values $a_{n}=k\left(\left\lceil n p_{1}\right\rceil-\left\lceil(n-1) p_{1}\right\rceil\right)$ for $n>0$ and $k=1$. Now replace the remaining 0 's in the sequence with 2's, by replacing $p_{1}$ with $p_{2} /\left(1-p_{1}\right)$ and incrementing $k$ to 2 , in which case the index $n$ on $a_{n}$ refers, naturally, to the $n$th zero of the preceding list. Continuation of this construction for $k=3,4, \cdots$ gives a real number:

$$
R=[0 ; 1,2,1,3,1,4,2,1,5,6,2,1,3,1,7,1,8,2,1,4,1,3,2,1,9,1, \ldots] .
$$

Through 100,000 elements the geometric and harmonic means for $R$ work out to be 2.6753 and 1.7454, respectively. Although we have not done so, it should be possible to prove for example that the limiting geometric mean of these elements is indeed $K_{0}$.

\section{Computation of Khintchine means}

As intimated in our introduction, calculation of $K_{0}$ has occupied the attention of various researchers. For example, Gosper recently computed $K_{0}$ to 2217 digits [12]. Our 7350-digit value is in complete agreement with Gosper's 2217 digits. There is also the interesting problem of computing fraction elements from decimal representations of certain real numbers, a task that one may wish to do in, say, statistical experiments involving Khintchine means. We mention that in [21] an interesting algorithm is presented for computation of fraction elements without recourse to decimal input. Instead, differential properties of an appropriate function are used. For example, Shiu resolved 10,000 elements of the fraction for $e^{\pi}$ using properties of the function $f(t)=\sin (\log (t))$.

By exploiting various modern algorithms to be described presently, the present authors have explicitly computedcdots $K_{0}$ and $K_{-1}$ to more than 7350 decimal digit accuracy. These computations were performed with the aid of the MPFUN multiprecision software $[4,5]$, which was found to be significantly faster for our purposes than other available multiprecision facilities. One utilizes this software by writing ordinary Fortran-90 code, with multiprecision variables declared to be of type mp_integer, mp_real or mp_complex. In the computations described below, the level of precision was sufficiently high that the "advanced" routines of the Fortran-90 MPFUN library were employed. These routines employ special algo- 
rithms, including fast Fourier transform (FFT) multiplication, which are efficient for extra-high levels of precision.

The constant $K_{0}$ was computed using the formula given above in Theorem 3, with the free integer parameter $N=100$, and with $N=120$ as a check. The implementation of this formula was straightforward except for the computation of the Riemann zeta function. To obtain 7350-digit accuracy in the final result, 2048 terms of the indicated series were evaluated, which requires $\{\zeta(2 k), 0 \leq k \leq 2048\}$ to be computed. One approach to compute these zeta function values is to apply formulas due to P. Borwein [8]. These formulas are very efficient for computing one or a few zeta function values, but when many values are required as in this case, another approach was found to be more efficient. This method is based on an observation that has previously been used in numerical approaches to Fermat's "Last Theorem" [9, 10], namely

$$
\begin{aligned}
\operatorname{coth}(\pi x) & =\frac{-2}{\pi x} \sum_{k=0}^{\infty} \zeta(2 k)(-1)^{k} x^{2 k} \\
& =\cosh (\pi x) / \sinh (\pi x) \\
& =\frac{1}{\pi x} \cdot \frac{1+(\pi x)^{2} / 2 !+(\pi x)^{4} / 4 !+(\pi x)^{6} / 6 !+\cdots}{1+(\pi x)^{2} / 3 !+(\pi x)^{4} / 5 !+(\pi x)^{6} / 7 !+\cdots} .
\end{aligned}
$$

Let $N(x)$ and $D(x)$ be the numerator and denominator polynomials obtained by truncating these two series to $n$ terms. Then the approximate reciprocal $Q(x)$ of $D(x)$ can be obtained by applying the Newton iteration

$$
Q_{k+1}(x):=Q_{k}(x)+\left[1-D(x) Q_{k}(x)\right] Q_{k}(x) .
$$

Once $Q(x)$ has been computed to sufficient accuracy, the quotient polynomial is simply the product $N(x) Q(x)$. The required values $\zeta(2 k)$ can then be obtained from the coefficients of this polynomial.

Computation time for the Newton iteration procedure can be reduced by starting with a modest polynomial length and precision level, iterating to convergence, doubling each, etc., until the final length and precision targets are achieved. Computation time can be further economized by performing the two polynomial multiplications indicated in the above formula using a FFT-based convolution scheme. In our implementation, FFTs were actually performed at two levels of this computation: (i) to multiply pairs of polynomials, where the data elements to be transformed are the multiprecision polynomial coefficients, and (ii) to multiply pairs of multiprecision numbers, where the data elements to be transformed are integers representing successive sections of the binary representations of the two multiprecision numbers.

The constant $K_{-1}$ was computed by applying the formula in Corollary 6 . Again, the challenge here is to precompute values of the Riemann zeta function for integer values. But in this case both odd and even values are required. The odd values can be economically computed by applying the following two formulas, the first given by Ramanujan, but simplified slightly and known earlier; the second derived by differentiating a companion identity of Ramanujan [6, ch. 14]: 


$$
\begin{aligned}
\zeta(4 N+3)= & -2 \sum_{k=1}^{\infty} \frac{1}{k^{4 N+3}(\exp (2 k \pi)-1)} \\
& -\pi(2 \pi)^{4 N+2} \sum_{k=0}^{2 N+2}(-1)^{k} \frac{B_{2 k} B_{4 N+4-2 k}}{(2 k) !(4 N+4-2 k) !}, \\
\zeta(4 N+1)= & -\frac{1}{N} \sum_{k=1}^{\infty} \frac{(2 \pi k+2 N) \exp (2 \pi k)-2 N}{k^{4 N+1}(\exp (2 k \pi)-1)^{2}} \\
& -\frac{1}{2 N} \pi(2 \pi)^{4 N} \sum_{k=1}^{2 N+1}(-1)^{k} \frac{B_{2 k} B_{4 N+2-2 k}}{(2 k-1) !(4 N+2-2 k) !} .
\end{aligned}
$$

Here $B_{2 k}$ is as always the $2 k$ th Bernoulli number.

Alternatively, the formulas can be written in terms of the even zetas as

$$
\begin{aligned}
\zeta(4 N+3)= & -2 \sum_{k=1}^{\infty} \frac{1}{k^{4 N+3}(\exp (2 k \pi)-1)} \\
& +\frac{1}{\pi}\left\{\frac{(4 N+7)}{2} \zeta(4 N+4)-\sum_{k=1}^{N} 2 \zeta(4 k) \zeta(4 N+4-4 k)\right\}, \\
\zeta(4 N+1)= & -\frac{1}{N} \sum_{k=1}^{\infty} \frac{(2 \pi k+2 N) \exp (2 \pi k)-2 N}{k^{4 N+1}(\exp (2 k \pi)-1)^{2}} \\
& +\frac{1}{2 N \pi}\left\{\sum_{k=1}^{2 N}(-1)^{k} 2 k \zeta(2 k) \zeta(4 N+2-2 k)+(2 N+1) \zeta(4 N+2)\right\} .
\end{aligned}
$$

These two formulas are not very economical for computing a single odd value or just a few odd values of $\zeta(k)$ - again, the formulas in [8] are more efficient for such purposes. But these Ramanujan formulas are quite efficient when a large number of odd zetas are required. Note that the infinite series in the two formulas can be inexpensively evaluated for many $N$ simultaneously, since the expensive parts of these expressions do not involve $N$. Further, the evaluation of the infinite series can be cut off once terms for a given $N$ are smaller than the "epsilon" of the numeric precision level being used. Happily, convergence here is fairly rapid for large $N$.

At first glance, the latter summations in these two formulas may appear quite expensive to evaluate. But note that each is merely the polynomial product of two vectors consisting principally of even zeta values. Thus, both sets of summation results can be computed using multiprecision FFT-based convolutions.

Computation of $K_{0}$ to 7350 digit precision required 2.5 hours on an IBM RS6000/ 590 workstation, and computation of $K_{-1}$ also to 7350 digits required some 12 hours. Excerpts of the resulting decimal expansions for each are included in the appendix. The complete expansions are available from the authors.

One intriguing question that was raised decades ago [27] is whether the continued fraction elements of $K_{0}$ themselves enjoy a limiting geometric mean $K_{0}$. We can of course ask more generally whether, for the fraction elements of any Khintchine mean $K_{p}$, the limiting Hölder mean of order $q$ is in fact $K_{q}$. During the task of computing from a given decimal representation a Hölder mean of some order, the issue of where to terminate the list of continued fraction elements is an interesting 
TABLE 1. Continued fraction statistics

\begin{tabular}{|l|r|l|l|}
\hline Constant & $\begin{array}{c}\text { Cont. Frac. } \\
\text { Elements }\end{array}$ & $\begin{array}{c}\text { Geometric } \\
\text { mean }\end{array}$ & $\begin{array}{c}\text { Harmonic } \\
\text { mean }\end{array}$ \\
\hline$K_{0}$ & 7182 & 2.660716 & 1.745541 \\
$K_{-1}$ & 7052 & 2.722471 & 1.746871 \\
$A$ & 15000 & 2.358251 & 1.745395 \\
$R$ & 100000 & 2.6753 & 1.7454 \\
$Z_{2}$ & 5206016 & 2.685482 & 1.7454074 \\
$\pi$ & 17001303 & 2.686393 & 1.745882 \\
\hline
\end{tabular}

one. We employed a simple criterion: if $x$ is known numerically, to $D$ decimals to the right of the decimal point, generate continued fraction elements for $x$ until a convergent $p / q$ has $2 q^{2}>10^{D}$. The motivation for choosing this simple criterion is the theorem that at least one of any two successive convergents must satisfy

$$
\left|\frac{p}{q}-x\right|<\frac{1}{2 q^{2}}
$$

and conversely, any reduced ratio $p / q$ satisfying this inequality must be a convergent of $x$ [13].

Our results are shown in Table 1, together with results for the constants $K_{-1}, A$, $R, Z_{2}$ (which were defined above), and $\pi$. To give statistical perspective to our results for $K_{0}$ and $K_{-1}$, we computed the geometric and harmonic means of the first 7000 fraction elements for each of 100 pseudorandom multiprecision numbers of the same precision, namely 7350 decimal digits. The average and standard deviation of their geometric means were 2.683740 and 0.030124 , respectively. The same statistics for their harmonic means were 1.745309 and 0.011148 , respectively. Note that these two averages are in good agreement with the theoretical values $K_{0}$ and $K_{-1}$. In any event, it appears that the geometric and harmonic means for the first 7182 elements of our 7350-digit $K_{0}$ are within reasonable statistical limits of the expected theoretical values.

A question implicitly asked in the previous section is whether $K_{0}$ or $K_{-1}$ is algebraic. This question can be numerically explored by means of integer relation algorithms. A vector of real numbers $\left(x_{1}, x_{2}, \ldots, x_{n}\right)$ is said to possess an integer relation if there exist integers $a_{k}$ such that $a_{1} x_{1}+a_{2} x_{2}+\cdots+a_{n} x_{n}=0$. It can easily be seen that a real number $\alpha$ is algebraic of degree $n-1$ if and only if the vector $\left(1, \alpha, \alpha^{2}, \ldots, \alpha^{n-1}\right)$ possesses an integer relation. Even if $\alpha$ is not algebraic, integer relation algorithms produce bounds that allow one to exclude relations within a region.

We employed the "PSLQ" algorithm developed by Ferguson and one of the authors, a simplified version of which is given in [3]. This algorithm, when applied to power vectors generated from our computed values of $K_{0}$ or $K_{-1}$, found no relations for either. On the contrary, we obtained the following result: if $K_{0}$ satisfies a polynomial of the form

$$
0=a_{0}+a_{1} \alpha+a_{2} \alpha^{2}+a_{3} \alpha^{3}+\cdots+a_{50} \alpha^{50}
$$

in the variable $\alpha$, then the magnitude of some integer coefficient $a_{k}$ exceeds $10^{70}$. The same was found to be true for $K_{-1}$. 
In a second experiment, we explored the possibility that $K_{0}$ or $K_{-1}$ is given by a multiplicative formula involving powers of primes and some well-known mathematical constants. To that end, let $p_{k}$ denote the $k$ th prime. We established, using PSLQ, that neither $K_{0}$ nor $K_{-1}$ satisfies a relation of the form

$$
\begin{aligned}
0= & a_{0} \log \alpha+\sum_{k=1}^{15} a_{k} \log p_{k} \\
& +a_{16} \log \pi+a_{17} \log e+a_{18} \log \gamma+a_{19} \log \zeta(3)+a_{20} \log \log 2
\end{aligned}
$$

with integer coefficients $a_{k}$ of absolute value $10^{20}$ or less. By exponentiating this expression, it follows that neither $K_{0}$ nor $K_{-1}$ satisfies a corresponding multiplicative formula with exponents of absolute value $10^{20}$ or less.

There are many other tests that might be applied. For example, further work might be to rule out the possibility that $\log K_{0}$, $\left(\log K_{0}\right)(\log 2)$, or one of many other forms involving $K_{0}$ be an algebraic number of low degree.

\section{ACKNOWLEDGMENT}

Thanks are due to Robert Corless, Greg Fee, Thomas Wieting, Simon Plouffe, and Joe Buhler for many helpful discussions. We are grateful to a referee for suggesting the construction of the candidate number $R$ at the end of $\S 4$.

\section{APPENDIX}

\section{The Khintchine Constant $K_{0}$ to 7,350 Digits}

$$
\begin{aligned}
& 2 . \\
& 68545200106530644530971483548179569382038229399446 \\
& 29530511523455572188595371520028011411749318476979 \\
& 95153465905288090082897677716410963051792533483259 \\
& 66838185231542133211949962603932852204481940961806 \\
& 86641664289308477880620360737053501033672633577289 \\
& 04990427070272345170262523702354581068631850103237 \\
& 46558037750264425248528694682341899491573066189872 \\
& 07994137235500057935736698933950879021244642075289 \\
& 74145914769301844905060179349938522547040420337798 \\
& 56398310157090222339100002207725096513324604444391 \\
& \ldots \ldots \\
& 36909874406573435125594396103980583983755664559601
\end{aligned}
$$

The Khintchine Harmonic Mean $K_{-1}$ to 7,350 Digits

1.

74540566240734686349459630968366106729493661877798 42565950137735160785752208734256520578864567832424 20977343982577985596531102601834294460206578713176 15026238960612981165718728271638949622593992929776 06160830078357479801549029312671643067241248453710 96077711207484391474195803753220015690822609477078 44894635568203493582068440202422591615018316479048 29229656977733143662210991806388842581650599997697 61391683577259217628635718712601565066754443340174 00283376465305136584406098398017126202832041200630 
.....

78553128249666473680304034761497467330708479436280

\begin{tabular}{cc}
\multicolumn{3}{c}{ Khintchine Means $K_{p}$ to 50 Digits for Various Negative $p$} \\
$p$ & $K_{p}$ \\
-2 & $1.450340328495630406052983076680697881408299979605904 \ldots$ \\
-3 & $1.313507078687985766717339447072786828158129861484792 \ldots$ \\
-4 & $1.236961809423730052626227244453422567420241131548937 \ldots$ \\
-5 & $1.189003926465513154062363732771403397386092512639671 \ldots$ \\
-6 & $1.156552374421514423152605998743410046840213070718761 \ldots$ \\
-7 & $1.133323363950865794910289694908868363599098282411797 \ldots$ \\
-8 & $1.115964408978716690619156419345349695769491182230400 \ldots$ \\
-9 & $1.102543136670728013836093402522568351022221284149318 \ldots$ \\
-10 & $1.091877041209612678276110979477638256493272651429656 \ldots$
\end{tabular}

\section{REFERENCES}

1. R. Adler, M. Keane and M. Smorodinsky, "A construction of a normal number for the continued fraction transformation," J. Number Theory 13 (1981), 95-105. MR 82k:10070

2. M. Abramowitz and I. A. Stegun, Handbook of mathematical functions, Dover Publications, Inc., New York, 1972. MR 94b:00012

3. D. H. Bailey, J. M. Borwein, and R. Girgensohn, "Experimental evaluation of Euler sums," Experimental Math. 3 (1994), 17-30. MR 96e:11168

4. D. H. Bailey, "A Fortran-90 based multiprecision system," ACM Trans. on Math. Software 21 (1995), 379-387.

5. _ "Multiprecision translation and execution of Fortran programs," ACM Trans. on Math. Software 19 (1993), 288-319.

This software and documentation, as well as that described in [4], may be obtained by sending electronic mail to mp-request@nas.nasa.gov, or by using Mosaic at address http://www.nas.nasa.gov.

6. B.C. Berndt, Ramanujan's notebooks, Part III, Springer Verlag, New York, 1991. MR 92j:01069

7. D. Borwein, J.M. Borwein, and R. Girgensohn, "Explicit evaluation of Euler sums," Proc. Edinburgh Math. Soc. 38 (1995), 277-294. MR 96f:11106

8. P. Borwein, "An efficient algorithm for the Riemann zeta function," submitted for publication. Available from http://www.cecm.sfu/ pborwein.

9. J. Buhler, R. Crandall, and R. Sompolski, "Irregular primes to one million," Math. Comp. $\mathbf{5 9}$ (1992), 717-722. MR 93a:11106

10. J. Buhler, R. Crandall, R. Ernvall, and T. Metsänkylä, "Irregular primes and cyclotomic invariants to four million," Math. Comp. 61 (1993), 151-153. MR 93k:11014

11. R. Corless, personal communication.

12. R. W. Gosper, personal communication.

13. A. Khintchine, Continued fractions, University of Chicago Press, Chicago, 1964.

14. D. Lehmer, "Note on an absolute constant of Khintchine," Amer. Math. Monthly 46 (1939), 148-152.

15. L. Lewin, Polylogarithms and associated functions, North Holland, New York, 1981. MR 83b:33019

16. H. Niederreiter, Random number generation and quasi-Monte-Carlo methods, CBMS-NSF Regional Conf. Ser. Appl. Math., vol. 63, 1992. MR 93h:65008

17. N. Nielsen, Die Gammafunktion, Princeton University Press, 1949.

18. S. Plouffe, personal communication.

19. C. Ryll-Nardzewski, "On the ergodic theorems (I,II)," Studia Math. 12 (1951) 65-79. MR 13:757a; MR 13:757b

20. D. Shanks and J. W. Wrench, "Khintchine's constant," Amer. Math. Monthly 66 (1959), 276-279. MR 21:1950

21. P. Shiu, "Computation of continued fractions without input values," Math. Comp. 64 (1995), 1307-1317. MR 96b:11165 
22. C.L. Siegel, Transcendental numbers, Chelsea, New York, 1965.

23. K. R. Stromberg, An introduction to classical real analysis, Wadsworth, Belmont, CA, 1981. MR 82c:26002

24. I. Vardi, Computational recreations in mathematica, Addison-Wesley, Redwood City, CA, 1991. MR 93e:00002

25. T. Wieting, personal communication.

26. J. W. Wrench, "Further evaluation of Khintchine's constant," Math. Comp. 14 (1960), 370371. MR 30:693

27. J. W. Wrench and D. Shanks, "Questions concerning Khintchine's constant and the efficient computation of regular continued fractions," Math. Comp. 20 (1966), 444-448.

28. D. Zagier, personal communication.

NASA Ames Research Center, Mail Stop T27A-1, Moffett Field, California 940351000

E-mail address: dbailey@nas.nasa.gov

Centre for Experimental and Constructive Mathematics, Simon Fraser University, Burnaby, British Columbia V5A 1S6, Canada

E-mail address: jborwein@cecm.sfu.ca

Center for Advanced Computation, Reed College, Portland, Oregon 97202

E-mail address: crandall@reed.edu 